\title{
ENCIERRO, PATOLOGÍA Y GÉNERO: LOS SUJETOS DE LA EXCLUSIÓN PSIQUIÁTRICA
}

\section{CONFINEMENT, PATHOLOGY AND GENDER: THE SUBJECTS OF PSYCHIATRIC EXCLUSION}

\author{
GRACIELA RAMÍREZ IGLESIAS ${ }^{1}$ \\ NATHALIA QUIROZ DEL POZO ${ }^{2}$
}

Recibido: 15 de mayo de 2017 Aceptado: 29 de agosto de 2017

\footnotetext{
${ }^{1}$ Pontificia Universidad Católica del Ecuador, Facultad de Psicología, Quito, Ecuador (gramirez522@puce.edu.ec).

${ }^{2}$ Pontificia Universidad Católica del Ecuador, Facultad de Psicología, Quito, Ecuador (naquiroz@puce.edu.ec).
} 



\section{ENCIERRO, PATOLOGÍA Y GÉNERO: LOS SUJETOS DE LA EXCLUSIÓN PSIQUIÁTRICA}

\section{CONFINEMENT, PATHOLOGY AND GENDER: THE SUBJECTS OF PSYCHIATRIC EXCLUSION}

Graciela Ramírez Iglesias y Nathalia Quiroz del Pozo

Palabras clave: hospital psiquiátrico, institución total, poder, violencia estructural,
estudios de género, sujeto.

Key words: psychiatric hospital, total institution, power, structural violence, gender studies, subject.

\section{RESUMEN}

Esta investigación basa su contenido en una problematización del internamiento prolongado o indefinido de mujeres dentro del Hospital Psiquiátrico. A partir de los datos obtenidos de la observación participante y continuada de una institución psiquiátrica quiteña en los períodos 2003-2007 y 2009-2010, se realiza un análisis desde las obras de
Erwing Gofman (1961), Michael Foucault (1992), Pierre Burgois (2005) y Johan Scott (1996). En este artículo se presentan algunos elementos que le permitan al lector la posibilidad de repensar al Hospital Psiquiátrico como un lugar posible, donde caduquen las prácticas arcaicas y donde se vuelva la mirada y la escucha hacia el sujeto. 


\section{ABSTRACT}

This research bases its content on a problematization of the long or indefinite internment of women inside the Psychiatric Hospital. Based on the data obtained from the participant and continuous observation of a psychiatric institution in the period 2003-2007 and 2009-2010, an analysis is made from the works of Erwing Gofman (1961), Michael
Foucault (1992), Pierre Burgois (2005) and Johan Scott (1996). This article presents some elements that allow the reader the possibility of re-thinking the Psychiatric Hospital as a possible place where archaic practices expire and where the look and the listening to the subject are returned.

\section{PREÁMBULO}

"Un acto de hospitalidad no puede ser sino poético" Derrida,1997

"El hospital psiquiátrico está lleno de silencios y batas blancas. Está lleno de pastillitas mágicas que espantan dolores y hasta hay unas máquinas que te transportan a otra dimensión. En el hospital hay espejos que ya no reflejan nada, se han transformado en cristales quebrados. En el hospital hay canciones, cuyas melodías son imperceptibles para los doctos que tienen el conocimiento en el maletín. En el hospital hay letras, en el hospital hay huellas, en el hospital circulan palabras que los oídos experimentados han preferido no escuchar. ¿Hay palabra en los locos', en los enfermos? ¡Pero esa palabra horroriza! ¡Esa imagen perturba! Esa palabra, misma que los sanos transformamos en estadísticas y diagnósticos. Esa palabra, que aquellos que están del lado estelar del escritorio prefieren silenciar para siempre gracias a la autorización que obtienen del representante legal, para legitimar el silencio. En los jardines

\footnotetext{
'La palabra "loco" ha sido usada peyorativamente para referirse a los enfermos mentales. El uso de esta palabra es estratégico en este trabajo y pretende evidenciar esa carga peyorativa. El psicoanálisis cuestiona a la noción de enfermedad mental desde una práctica que da prioridad a la palabra del paciente y no al diagnóstico otorgado por los manuales especializados. Para el psicoanálisis la noción de locura es claramente cuestionable.
} 
del hospital se pasan por alto historias cuyos protagonistas no están muertos, pero cuya suerte no es envidiable por ningún vivo. Vivísimos los sanos, nos construimos un espacio amurallado para que los que nos recuerdan que la cordura es una línea frágil, estén bien guardados y callados. La palabra trans- forma esos cristales quebrados en imagen viva de un sujeto, que seguro tiene algo que decir, la palabra es esa melodía que se desliza entre la queja de los desterrados, la palabra es la huella indeleble de que ahí existen sujetos, no cuerpos vacíos silenciados con las pastillas mágicas y el "electroshock".2

\section{INTRODUCCIÓN}

El presente trabajo está dividido en tres secciones: en la primera se describe brevemente el método utilizado para recolectar la información analizada. Se ha decidido partir de la información recolectada a través de la observación participante dentro de una institución psiquiátrica de la ciudad de Quito (realizada en los períodos julio 2004-marzo 2008 y diciembre 2009junio 2010). Este tipo de metodología ha sido utilizada en varias disciplinas como la antropología, la sociología y la psicología, como un instrumento en la investigación cualitativa para recoger datos sobre la gente, los procesos y las culturas. Como herramienta investigativa es definida por Marshall y Rossman (1989) como "la descripción sistemática de eventos, comportamientos y artefac- tos en el escenario social elegido para ser estudiado" (p. 79).

En la segunda sección se resumen algunos hitos de la historia de la psiquiatría en el mundo y algunas anotaciones sobre las particularidades en el Ecuador. Paulatinamente, se presenta información que evidencia las lógicas de poder en las que están sostenidas estas instituciones y como echaron cimientos hasta llegar a nuestros días. Se analizarán los datos obtenidos en los acápites anteriores, introduciendo cuatro conceptos: el de Institución Total de Erwing Gofman (1961), el de poder de Michael Foucault (1992), el de violencia simbólica de Pierre Burgois (2005) y el de género como elemento constitutivo de las relaciones sociales de Johan Scott (1996).

\footnotetext{
${ }^{2}$ Esta carta fue escrita como apoyo a un editorial de autoría del Dr. Iván Sandoval publicado en el Diario "El Universo", Guayaquil,1 de febrero de 2011. Recuperado de http://www.eluniverso.com/2011/02/01/1/1363/ efrain-descansa-paz.html.
} 
Finalmente, en la tercera sección, a manera de conclusiones, se evidenciarán los elementos estructurales de la institución psiquiátrica que derivan en formas de violencia que atentan contra los derechos humanos de las usuarias de dichos servicios. No se desconoce que los usuarios estén expuestos también a vul- neraciones en sus derechos; sin embargo, este artículo se enfocará en las motivaciones de los internamientos prolongados de mujeres en la institución psiquiátrica; para a través del enfoque de género, dilucidar de qué manera las mujeres se ven afectadas de una forma particular por la visión psiquiátrica tradicional.

\section{CONTEXTO Y MÉTODO}

El análisis de dispositivos tan arraigados como los que se dan en el hospital psiquiátrico, requiere una observación exhaustiva como camino para leer los deslices y las diferencias en las dinámicas institucionales monótonas que ocultan tras de sí otros sentidos, como decía (Lévi-Strauss, 1979) "La intención exhaustiva que inspira nuestras investigaciones transforma en muy gran medida su objeto. Técnicas tomadas aisladamente pueden aparecer como algo dado en bruto, legado histórico o resultado de una componenda entre las necesidades del hombre y los constreñimientos del medio" (p. 16).

A través de la observación participante dentro de una institución psiquiá- trica de la ciudad de Quito ${ }^{3}$ se busca hacer una comparación entre las promesas de salud de la psiquiatría y lo que es el vivir cotidiano de las pacientes internadas. Es necesario señalar, que la permanencia tan prolongada de una de las investigadoras en la institución psiquiátrica (tres años ocho meses en el primer período ${ }^{4}$ ) se debió a que formó parte desde el año 2004, de un grupo de investigación in-situ a cargo del Dr. Ivan Sandoval $\left.\right|^{5}$, el mismo estaba asociado a las actividades de la Escuela Freudiana del Ecuador y trataba de la revisión del Seminario 3 de Jacques Lacan "Las Psicosis" (1955-1956).

Basados en el trabajo de Haraway (1995) quien propone que: "solamente una perspectiva parcial promete una vi-

\footnotetext{
${ }^{3}$ Se ha decidido conservar el anonimato de la institución en la que se realizó la observación basados en consideraciones de confidencialidad.

${ }^{4}$ Los seis meses de observación en el período 2009-2010 fue gestión personas de una de las investigadoras con el Director de la institución.
}

${ }^{5}$ Médico Psiquiatra y Psicoanalista, miembro del grupo ABCdario Freud Lacan 
sión objetiva" (p.329), se puede sostener que el conocimiento situado, nos permite inferir que desde diferentes posiciones de sujeto se perciben diferentes realidades. La observación participante permite al investigador ser parte del estudio de una forma activa. Según De Walt \& De Walt (2002) el trabajo de campo involucra mirada activa, una memoria cada vez mejor, entrevistas informales, escribir notas de campo detalladas, y, tal vez lo más importante, paciencia. La observación participante permite a los investigadores aprender acerca de las actividades de las personas en estudio en el escenario natural, Schensul y Lecompte (1999) hablan de "el proceso de aprendizaje a través de la exposición y el involucrarse en el día a día o las actividades de rutina de los participantes en el escenario del investigador" (p.91)

Bernard (1994) define a este método como el proceso para establecer relación con una comunidad y aprender a actuar al punto de mezclarse, de for- ma que sus miembros actúen de forma natural; y luego salirse de la comunidad para sumergirse en los datos y comprender lo que está ocurriendo. La observación participante se caracteriza por acciones tales como tener una actitud abierta, libre de juicios, estar interesado en aprender más acerca de los otros, ser consciente de la propensión a cometer errores, la mayoría de los cuales pueden ser superados (De Walt y De Walt, 2002).

Schensul, Schensul, \& Lecompte (2002) consideran tres razones relevantes para usar observación participante en la investigación: identificar y guiar relaciones con los informantes; ayudar al investigador a sentir cómo están organizadas y priorizadas las cosas, cómo se interrelaciona la gente, y cuáles son los parámetros culturales; mostrar al investigador lo que los miembros de la cultura estiman que es importante en cuanto a comportamientos, liderazgo, política, interacción social y tabúes.

\section{BREVE RECORRIDO HISTÓRICO DE LA PSIQUIATRÎA: LOS ALCANCES PARA EL ECUADOR}

La clásica intervención para los enfermos mentales ha sido históricamente la hospitalización, como dice Michel Foucault en su texto sobre el poder psiquiátrico: "El hospital, durante mucho tiempo siguió siendo un lugar ambiguo: de constatación para una verdad oculta y de prueba para una verdad por producir" (Foucault, 1974, p.383). La verdad oculta es la verdad de la patología, de la 
que solo pueden hablar los que manejan el lenguaje médico; para el resto de personas este lenguaje es un código que ubica al sujeto en un lugar de sumisión ante el médico. Foucault a lo largo de su obra, centra la mirada en las relaciones de poder que se pueden ubicar en distintos tipos de instituciones. Dentro de su Historia de la locura en la época clásica (1973) podemos situar tres momentos importantes en el devenir de esta ciencia. El primero de estos está ligado con la moral y la magia. Un enfermo mental en la edad media era nombrado y tratado como un endemoniado. Estar frente a la enfermedad mental era motivo para pensar en la posesión demoníaca o en la magia negra. Dado que la religión tenía un peso infinito en el discurso de la ciencia, el cuerpo y la mente eran entendidos como inexorables. Científicamente, del alma no se podía decir nada ya que no había medios para explorarla y el comportamiento humano estaba determinado en cada caso por designios divinos.

El segundo momento relevante de la psiquiatría se observa en el renacimiento, donde decayó la idea que todos los males del mundo eran causa divina y la enfermedad mental fue considerada por primera vez una consecuencia del ambiente. Aún no se podía contar con clasificaciones diagnósticas detalladas, pero un científico llamado Paracelso ${ }^{6}$ (1493-1541) dio cuenta ya de las diferencias y particularidades de la enfermedad mental. Paracelso en su tratado "Sobre las enfermedades que privan de la razón" dividió a estas patologías aún desconocidas en cinco grupos: epilepsia, manía, locura verdadera, baile de San Vito y "suffocatio intellectus". La locura verdadera se divide, según este autor en: lunáticos, insanos, vesánicos, melancólicos y obsesos. De ahí en adelante la clínica psiquiátrica ha producido un interminable conjunto de clasificaciones para las enfermedades, con más detalle de los síntomas que identifican a estos malestares, de tal suerte que muchos más sujetos puedan calzar con estas clasificaciones.

Tenemos en la obra del psiquiatra francés Henry Ey denominada Naissance de la Médecine (1981), un tratado de psiquiatría clínica y terapéutica en el cual menciona que el nacimiento de la asistencia a los enfermos mentales se da en la Edad Media en los países musulmanes en el siglo XV y posteriormente, tras la fundación en 1571 de la orden de los hermanos de la Caridad por San Juan de Dios, se difunde en Europa y en Hispanoamérica. En un inicio los mendigos, los soldados desertores, los indigentes, son expulsados de las ciudades pues en ellos está la fuente de todo mal; es una muchedumbre

\footnotetext{
${ }^{6}$ Alquimista, médico y astrólogo suizo
} 
que puede ir de una ciudad a otra, que no tienen un lugar de estadía. En esta situación en 1656, se prohíbe en Francia la mendicidad, so pena de látigo y reclusión sin distinción de edades o de sexos, de enfermedades o de condiciones. Los militares en recogen a los que no han asumido la orden y los asila en un espacio que se llamará en ese momento Hospital General. Cuatro años más tarde se tiene informe que solamente en Francia en el asilo de la Salpetriêre, se encuentran recluidos 1.460 mujeres y niños de tierna edad y en Bicetre 1.615 hombres adultos. En total en todos los asilos se internan entre $5.000 \mathrm{y}$ 6.000 personas, hecho que se extiende a toda Europa hacia el siglo XVII (Foucault, 1967, p.95). Así nacieron los primeros asilos para ser hogares que luchan contra "la pobreza y el vicio".

Hacia el siguiente siglo se fundan en toda Europa casas asistenciales herederas de los leprosarios y se instituye por ley la creación de asilos que son dados por la conversión de los bienes eclesiásticos para obras hospitalarias; un ejemplo es el que encontramos en un decreto de la Reforma que recomienda no abandonar las obras y darles la importancia de bienes del Estado. Es una preocupación del Estado y un deber de este cuidar a los enfermos y ya no solamente una obra de la Iglesia, que era parte del ejercicio de la caridad. El loco antes de ser considerado un objeto de conocimiento y de piedad se convierte en un sujeto moral. Esto creó lo que Foucault menciona en su Historia de la locura, como el "gran encierro" y como "el nacimiento del asilo" (Foucault, 1973, p.125).

En el siglo XVIII, tenemos a Phillipe Pinel (1745-1826), quien fue un médico francés que profundizó sobre el estudio de las llamadas enfermedades mentales y fue considerado por muchos el primer verdadero psiquiatra (Ackerknecht, 1993, p, 49). Podemos evidenciar que la clínica de Pinel basaba su estudio en la observación y el análisis detallado de los síntomas que se presentan a nivel de los fenómenos, perceptibles únicamente por el ojo experimentado. Tenemos entonces una constancia histórica que la medicina es una ciencia clasificadora; como afirma Braunstein, psiquiatra y psicoanalista contemporáneo: La medicina clasificadora es la del siglo XVIII la que precede al método anatomo-clínica. Su pretensión es la de asilar las enfermedades como especies naturales e incluirlas en un espacio clasificatorio homogéneo. Su modelo es la clasificación de las especies vegetales efectuada por Lineo. Su método es la descripción de los fenómenos tal como ellos se aparecen a los órganos de los sentidos, muy particularmente de la vista (Braunstein, 1980, p.14).

Según Braunstein podemos pensar que la clínica que inaugura Pinel, a pesar de ser un intento que la psiquia- 
tría sea una práctica más humanizada, es una ciencia que trata solamente sobre la mirada que recae en los síntomas del individuo-objeto y deja de lado la escucha del malestar que es lo que permitiría pasar de la concepción del individuo sano o loco de las ciencias positivistas hacia el sujeto del lenguaje que experimenta malestares que no necesariamente son observables. Sin embargo, es notable citar que uno de los logros más importantes de Pinel en lo que al tratamiento mental se trata, es que liberó a los alienados de las amarras que los mantenían sujetados a las paredes de sus habitaciones. Pinel insistió en que debía considerarse como necesaria la formación de profesionales especializados para atender a los alienados. Posteriormente, tenemos a Etienne Esquirol (1782-1840) quien se formó bajo los preceptos de la terapia moral de Pinel y consiguió promulgar una ley en 1838 que obligaba a la administración francesa a disponer de un asilo para dementes en cada departamento del país.

Otro momento a destacar es el siglo XIX, cuando el hospicio se transforma en hospital psiquiátrico. Se produce el espacio ideal para que la psiquiatría pueda establecer criterios de especialización para el abordaje clínico, criterios que solamente los médicos están autorizados a cuestionar. Emil Kraepelin, que es uno de los representantes más im- portantes de la psiquiatría del siglo XIX (1856-1926) se centró en la clínica como herramienta diagnóstica; su influencia produjo que la psiquiatría se transforme de una ciencia de la alienación a una especialidad médica. Las clasificaciones propuestas por este autor siguen siendo los criterios bajo los que se han desarroIlado las tipologías diagnósticas actuales: del DSM-IV, DSM-V y del CIE10.

Podemos entender aquí, que la terapéutica de la locura es el arte de subyugar y domesticar al otro, poniéndolo bajo la estricta dependencia de un hombre que por sus insignias científico-morales está autorizado a modificar sus ideas. Pinel en 1973 encabezando la famosa liberación de los enfermos mentales de sus cadenas en el hospital de Bicêtre, instala la escena fundadora de la psiquiatría moderna, donde esta se transforma en una forma de aislamiento apoyada ya no en tratamientos físicos sino en un llamado "tratamiento moral" con una mejor aceptación que los instrumentos de contención.

Finalmente, ya en el siglo XX, Eugen Bleuler, introdujo en 1911 el término esquizofrenia (Kaplan, 1994, P.205), a partir de esto, nacen los manuales estadísticos, los mismos que siguen las descripciones hechas por observadores clínicos; sin embargo el método usado para la clasificación y la descripción de las enfermedades tendría como efecto 
reproducir patrones que sean compartidos como un saber dentro de un grupo.

Mientras en Francia se consolidaba una práctica basada en la clasificación rígida de las enfermedades, en América Latina heredábamos la concepción de que el espacio de tratamiento para la enfermedad mental debía basar su diseño en la figura del hospicio. Es así como en 1785 se construye en la ciudad de Quito, en la calle que ahora conocemos como Ambato, el cuarto hospital psiquiátrico de América Latina: "El hospicio de Jesús, María y José", que posteriormente se transformaría en el "Hospital Psiquiátrico San Lázaro" que es actualmente la institución con estas características más antigua de América. En el acta de fundación se anota que fue creado para albergar locos, mendigos y prostitutas, "demandan inspección robusta los maniáticos, los alcohólicos y las mujeres de vida aireada" (Landázuri, 2008:40). Sin embargo, al poco tiempo se asilaron también a enfermos con lepra, y es precisamente es a ellos a quienes se dieron los primeros tratamientos de que se tiene noticias (López Cifuentes, 2007).

Como podemos ver, el marco en el que se funda la institución mental en el Ecuador es el mismo, el de albergar a las personas que se consideran un desecho. ¿Dónde caben los locos, en la esfera pública o en la privada? Lastimosamente se ha llegado a pensar que caben en la esfera perdida, muchos de los que ingresaron a los hospitales psiquiátricos pueden bien ubicarse entre los desaparecidos. Ciertos internos de la institución psiquiátrica llegan a tener internamientos de por vida porque la institución los absorbe a ellos y asusta a sus familias. La institución no se creó para tratarlos, se creó para albergarlos (López Cifuentes, 2007).

Además, la psiquiatría en el Ecuador tardó mucho en situarse como ciencia necesaria dentro de las facultades y hasta hoy sigue siendo un tema donde no muchos quieren verse concernidos. Situemos en cómo se instauró la cátedra de psiquiatría: en 1912 en Ecuador se inicia la cátedra de psiquiatría en la Universidad Central como materia de información elemental, los alumnos no estabas obligados a rendir exámenes sobre la misma. El primer titular de la cátedra es el Dr. Carlos Alberto Arteta García que, a su vez es el Director Médico del manicomio, a quien luego de un corto interinazgo ejercido por el Dr. Alberto Correa, remplazará el Dr. Julio Endara Moreno, quien a su vez es nombrado director del manicomio en 1924, cargo que lo mantiene por el espacio de un año y se convierte en profesor de neurología y psiquiatría en la Universidad Central, quien logra incorporar a estas materias dentro del pensum de estudio de Medicina con el carácter de obligatorias. 
Como podemos ver, la cátedra de medicina en Ecuador es tierna versus el nacimiento del hospicio, desde 1785 hasta 1912 no hubo una teoría que sostuviera mal o bien las prácticas que se daban dentro de este espacio. Es por eso que es tan difícil poder afirmar que la psiquiatría es la ciencia en cuyas prescripciones se incluye la posibilidad del internamiento. El hospicio ya estaba ahí tanto en el Ecuador como en el resto del mundo, es más viejo y más poderoso que la misma psiquiatría y son sus lógicas arraigadas de encierro las que han moldeado la psiquiatría a su conveniencia. Lastimosamente, el nacimiento de la psiquiatría lo que hizo es transformar las cadenas de los enfermos del hospicio en cadenas farmacológicas.

Apenas en los años setenta se realiza la fundación del Hospital "Sagrado Corazón de Jesús", un espacio que acogía inicialmente solo a pacientes femeninas, bajo una lógica completamente medicamentosa que hace alusión a los conceptos de esa época: En los años setenta la prescripción de antipsicóticos, antidepresivos tricíclicos, ansiolíticos, anticomiciales, etc., comercializados en el país, era la práctica cotidiana por parte de los médicos de esta casa.

Desde 1940, el Dr. Fernando Cazares de la Torre, como un Pinel de la psiquiatría ecuatoriana, lucha para que los pacientes no sean sometidos a reclusión, sujeción con sogas y otros maltratos. Se comienzan a usar algunos barbitúricos (secobarbital, amobarbital) y también la reserpina. Además, inicia la aplicación de choques con Cardiazol. A su retorno, en 1944, del Hospital psiquiátrico "St. Elizabeth", en Michigan, trae como novedad el uso del "electroshock", reforzado posteriormente por los conocimientos adquiridos por el Profesor Jorge Escudero M. en el Hospital "Santa Ana" en París. En la Institución quiteña el "electroshock" es una práctica constante desde los años cincuenta y no se aplica bajo parámetros médicos (López Cifuentes, 2007).

\section{LAS INSTITUCIONES TOTALES: CONCEPTO Y ALCANCES DESDE GOFFMAN}

Según Goffman 1961 "Toda institución absorbe parte del tiempo y del interés de sus miembros y les proporciona en cierto modo un mundo propio; tiene, en síntesis, tendencias absorbentes" (p.17). Si las instituciones tienden a absorber a sus miembros tienden también a anular subjetividades. Las instituciones totales a más de anular subjetividades, perennizan estructuras incuestionables. 
Entre las instituciones totales tenemos a aquellas que contienen a las personas que voluntaria o involuntariamente amenazarían a la sociedad.

Las cárceles y las correccionales contienen a los que voluntariamente intimidan a las personas de bien. En cambio, los hospicios, los hospitales psiquiátricos y los orfanatos albergan a esos sujetos que involuntariamente suponen peligro para otros. ¿Por qué las lógicas de estos dos tipos de instituciones son las mismas si sus poblaciones son definitivamente distintas? En ambos casos el internamiento involuntario termina por ex-cluir definitivamente a los sujetos de la sociedad.

Las instituciones totales son cerradas, hay un posicionamiento no escogido en la distribución del poder para muchos sujetos a los que no les será permitido nunca más cuestionar las prácticas de desajuste, deslocalización y desaparición de toda subjetividad. Goffman (1961) introduce características detalladas y específicas de las instituciones totales y los hospitales psiquiátricos se apegan oportunamente a ellas. "La característica central de las instituciones totales puede describirse como una ruptura de las barreras que separan de ordinario estos tres ámbitos de la vida. Primero, todos los aspectos de la vida se desarrollan en el mismo lugar y bajo la misma autoridad única. Segundo, cada etapa de la actividad diaria del miembro se lleva a cabo en la compañía inmediata de un gran número de otros, a quienes se da el mismo trato y de quienes se requiere que hagan juntos las mismas cosas. Tercero, todas las etapas de las actividades diarias están estrictamente programadas, de modo que una actividad conduce en un momento prefijado a la siguiente y toda la secuencia de actividades se impone desde arriba, mediante un sistema de normas formales explícitas y un cuerpo de funcionarios. Finalmente, las diversas actividades obligatorias se integran en un solo plan racional, deliberadamente concebido para el logro de los objetivos propios de la institución" (p. 20).

Las instituciones totales no están llamadas a preservar las características particulares de los sujetos, están destinadas a mantener las lógicas clásicas de la institución ${ }^{7}$, así como a perpetuar la distribución de poder en una estructura jerárquica que, a simple vista, parece imposible de romper. Según Goffman, las instituciones psiquiátricas no valen para lo que fueron concebidas; el internamiento, con sus características y consecuencias, es más un método de

\footnotetext{
${ }^{7}$ Con lógicas clásicas me refiero a la concepción foucaultiana que a pesar que los leprosarios han evolucionado en hospitales psiquiátricos, cárceles y hospicios; estos han conservado la misma estructura que convierte a ciertos sujetos en despojos de la sociedad.
} 
desajuste que de ajuste y organización del yo del paciente (1961), pareciera que lo que les interesa a estas instituciones es que los sujetos luzcan como una masa homogénea bien adaptada y que no cuestione las indicaciones de las autoridades.

\section{EL CONCEPTO DE PODER Y LAS INSTITUCIONES}

Foucault (1992) propone que: "Meter a alguien en prisión, encerrarlo, privarlo de comida, de calefacción, impedirle salir, hacer el amor, etc., ahí está la manifestación del poder más delirante que se pueda imaginar" (p.86). En los hospitales psiquiátricos se pueden observar privaciones similares a las de las cárceles y muchas veces los internamientos se vuelven condenas más extensas que las de los criminales. ¿Cuánto tiempo debería pasar un enfermo mental internado para que dicho internamiento no pierda sus cualidades terapéuticas y se convierta en un encarcelamiento? Como dice Foucault: "es menester aplicar un método perturbador, quebrar el espasmo mediante el espasmo (...) Hay que subyugar la totalidad del carácter de ciertos enfermos, vencer sus pretensiones, domesticar su arrebato, quebrantar su orgullo, mientras que es preciso excitar, alentar a otros..." (Foucault, 1974, p. 388).

Pensando en Foucault, nacen las preguntas ¿Cómo se estructura la economía del poder dentro de la institución psiquiátrica?, ¿Qué impacto diferencial tiene el internamiento prolongado en los hombres y las mujeres?, ¿Qué disparidades encontramos en los diagnósticos específicos que reciben las mujeres y que reciben los hombres? ¿Cuáles serían los posibles caminos que permitan desmontar las lógicas de poder existente en este espacio? ¿Por qué la institución psiquiátrica se ha convertido en una herramienta de la ex-clusión?

El sujeto de deseo, ese que habla no solamente con la voz sino a través del cuerpo, es silenciado en el hospital por la farmacología y el convencimiento absoluto que le han impuesto la pérdida de sus derechos como ciudadano. Cuando un enfermo recibe un diagnóstico grave, al entender como grave que sus síntomas requieren internamiento, este sujeto se transforma en un interdicto, es decir, se le retira el derecho a la palabra así como también el derecho que tiene como ciudadano para manejar sus cuentas bancarias, tener una pareja, salir a la calle libremente, entre otros. ¿Esta interdicción es parte del mecanismo que intenta producir un sujeto homogéneo 
que la estructura hospitalaria pueda manejar? Sujetos sin Voz, sin pertenencia étnica, sin representación política y sin género.

\section{ENFOQUE DE GÉNERO CONSTITUTIVO DE LAS RELACIONES SOCIALES: LA LECTURA DESDE LA VIOLENCIA ESTRUCTURAL}

El enfoque de género permite evidenciar las construcciones culturales y la creación de roles estereotipados para mujeres y hombres que a su vez han determinado una aparente dicotomía entre los sexos. Esta dicotomía se caracteriza por una inequitativa distribución de poder que se evidencia tanto en la esfera pública como en la privada en distintas formas de violencia, maltrato y discriminación a aquellas personas, hombres o mujeres que no cumplen con los roles supuestos para su sexo, Joan Scott afirma que: "el género más allá de una categoría útil para el análisis histórico, se ha convertido en una categoría necesaria para el diseño y aplicación de la política pública nacional y local dirigida a Grupos de Atención Prioritaria, porque ofrece un modo de diferenciar la práctica sexual de los roles sociales asignados a mujeres y hombres (Scott, 1996, p.1). En este punto es necesario situar del concepto propuesto por Burgois sobre violencia estructural: "La violencia estructural se refiere a la organización económico-política de la sociedad que impone condiciones de sufrimiento físico $\mathrm{y} / \mathrm{o}$ emocional, desde los altos índices de morbosidad y mortalidad hasta la pobreza y condiciones de trabajo abusivas y precarias" (Burgeois, 2005, p.13). Esta situación estructural de violencia involucra directamente a las instituciones del Estado y la institución psiquiátrica no se escapa; está estructuralmente asumido que los locos y las locas tienen que ser aislados y anulados de la vida comunitaria, esto se puede considerar una violencia directamente relacionada con condiciones de pobreza extrema en las casas de salud y con pocas o nulas posibilidades de una vida laboral productiva.

¿Qué se puede decir desde el discurso de género sobre la violencia que sufren las mujeres en los sistemas de salud mental cuando reciben diagnósticos, errados y pasan largos años internadas, porque el mismo sistema de salud no facilita la reinserción a sus familias y al ámbito laboral?

La diferencia de género es utilizada para producir estereotipos, en el 
caso de las instituciones psiquiátricas es paradojal, la diferencia sólo se pone en juego al momento de diagnosticar estereotipadamente a la mujer o al hombre "generalmente los diagnósticos que reciben las mujeres están relacionados con trastornos afectivos, crisis de ansiedad, depresiones y trastornos bipolares; en cambio los diagnósticos de los hombres en su mayoría tratan de alcoholismo, trastornos psicóticos en general, depresiones y trastornos bipolares" ${ }^{\prime \prime}$.

\section{CONCLUSIONES: LA MIRADA DEL GÉNERO EN LA INSTITUCIÓN MENTAL}

Los diagnósticos de las mujeres son influenciados directamente por el sistema de control de la sexualidad en el que se enmarca la sociedad y generan una brecha diferencial de diagnóstico y una eliminación de las características femeninas de las internas, debido a que son obligadas a renunciar definitivamente a las prácticas que les recuerde algo de su deseo.

La sexualidad es el meollo del asunto y es tomada como pretexto para normar el comportamiento no solo de las internas de la institución psiquiátrica sino también de las que no queremos ser futuras internas de la misma. Desde esta perspectiva la institución psiquiátrica se vuelve un mecanismo, como tantos otros de control para el "adecuado" comportamiento sexual.

Los testimonios de las mujeres escuchados para la realización de este trabajo posicionan al encierro y la exclusión como la primera alternativa de tratamiento, pero solo en el caso de las mujeres, estos son autorizados por sus mismas parejas o por sus padres porque su vida sexual se considera "reprobable". Pudimos conocer a mujeres que podrían ser partícipes de otro tipo de tratamientos que no necesariamente se basen en un discurso tradicional y moral que norma la sexualidad.

En la historia de la institución psiquiátrica encontramos las evidencias que este tipo de instituciones no fueron pensadas con fines terapéuticos sino con fines de encierro y aislamiento. No se pueden desconocer las bases en las que fueron creadas ya que su herencia se manifiesta hasta nuestros días.

Nuestro país no es la excepción, pudimos ver como una naciente psiquiatría se asentó en el Ecuador sin

\footnotetext{
${ }^{8}$ Información extraída de una entrevista realizada en Quito el 17 de mayo de 2010, al Dr. Giovanny Toscano, que trabajó en una institución psiquiátrica quiteña durante los períodos 1991-1996 y 2003-2008.
} 
mucha discusión y como la institución psiquiátrica actual es una muestra que hay instituciones que sobreviven como incuestionables. Los diagnósticos de las mujeres y hombres son afectados directamente por el sistema de sexo-género en el que se enmarca la sociedad y generan una brecha diferencial de diagnóstico y una eliminación de las características femeninas de las internas, debido a que son obligadas a renunciar definitivamente a todos los instrumentos y prácticas que les recuerde algo de su deseo como mujeres, pensando al deseo como un aspecto integral, más allá de la pura sexualidad humana.

La perspectiva que se mostró a lo largo de este trabajo pretende regresar la mirada al sujeto que se encuentra dentro de la institución psiquiátrica y pretende sensibilizar a operarios de salud sobre las formas alternativas de tratamiento para las llamadas enfermas mentales que no involucren a la anulación subjetiva que producen los tratamientos tradicionales. El pensar estructural y posicionalmente se ha convertido en una herramienta necesaria para el abordaje de las distintas patologías que se presentan en la clínica a través de una intervención desde una mirada que parte del análisis social y no solamente de las ciencias médicas.

La sexualidad es el meollo del asunto y es tomada como pretexto para normar el comportamiento no solo de las internas de la institución psiquiátrica, sino también de los que no queremos ser futuros internos de la misma. Desde esta perspectiva la institución psiquiátrica se vuelve un mecanismo, como tantos otros de control para el "adecuado" comportamiento sexual. Detrás del encierro de la mujer está un juicio moral que parte de esa necesidad de pensar a la mujer desde el "deber ser"; se espera que las mujeres personificaran la pureza y carecieran de deseo sexual.

Se ha considerado históricamente que la feminidad condiciona el padecimiento de determinadas patologías al cargar a las mujeres con el estigma de la llamada locura según asuman o rechacen los papeles que la organización social les impone. Las intervenciones que para las mujeres ha generado el saber psiquiátrico, legitiman la invisibilidad dentro de lo que sería una política de dominación y control, pero también de complicidad desde los estándares morales, revelan la necesidad del análisis de la llamada locura femenina para demostrar las contradicciones y las paradojas presentes en el inexistente concepto de salud mental.

Una corriente crítica a nivel mundial ha generado movimientos de transformación de la atención de la "salud mental" que apuntan a la introducción de toda una estructura comunitaria en la atención. Los puntales más fuertes de 
este proceso se centraron en la instauración de la modalidad de internaciones breves, a las que se apela como recurso de última instancia, y en la puesta en marcha de diversos dispositivos de integración (MSP, 2014). No se está hablando del cierre de los hospitales, ni en dejar a los internos en la calle o a los trabajadores de la salud pública sin sus puestos de trabajo. La cuestión es pensar en dejar a un lado el excesivo uso de fármacos y el encierro. De esta manera fomentamos la integración de los y las internas así como contribuimos a la disminución de una forma más de violencia contra las mujeres que ha sido naturalizada por la sociedad. 


\section{BIBLIOGRAFÎA}

Ackerknecht, E. (1993) Breve historia de la psiquiatría Buenos Aires: Guada litografía.

Bernard, H. Russell (1994). Research methods in anthropology: qualitative and quantitative approaches (segunda edición) Walnut Creek, CA: AltaMira Press.

Braunstein, N. (2008), Memoria la inventora, México: Siglo XXI,

Burgois, P. (2005) Más allá de una pornografía de la violencia. Lecciones desde El Salvador. En Ferrándiz, Francisco y Carles Feixa (eds). Jóvenes sin tregua. Culturas y políticas de la violencia. Barcelona: Anthropos.

Derrida, J. (1968) La Différance, Conferencia pronunciada en la Sociedad Francesa de Filosofía, publicada simultáneamente en el Bulletin de la Societé française de philosophie (julio-septiembre, 1968) y en Theorie Paidós (col. Quel, Ed. De Seuil, 1968).

Derrida, J. (2006) La hospitalidad, Buenos Aires: Ediciones de la flor.

DeWalt, K. M. \& DeWalt, B. R. (2002). Participant observation: a guide for fieldworkers. Walnut Creek, CA: AltaMira Press.

Ey, H. (1981) Naissance de la médecine: Paris, Masson.
Foucault, M. (1967). De los espacios otros. Conferencia dictada en el Cercle des études architecturals, 14 de marzo de 1967, publicada en Architecture, Mouvement, Continuité, n 5, octubre de 1984. Traducida por Pablo Blitstein y Tadeo Lima.

Foucault, M. (1973) Historia de la locura en la época clásica I y II, México: Fondo de cultura económica.

Foucault, M. (1974), El poder Psiquiátrico, Buenos Aires: Fondo de cultura económica.

Foucault, M. (1992), Microfísica del poder, Madrid: Las ediciones de la Piqueta.

Goffman, E, (1972) Internados: Ensayos sobre la situación social de los enfermos mentales, Buenos Aires: Amorrortu Editores.

Haraway, D. (1991) Ciencia, cyborg y mujeres, la reinvención de la naturaleza, Barcelona: Cátedra Feminismos.

Kaplan, H. \& Sadock, B. (1994) Compendio de psiquiatría. Barcelona España: Editorial Salvat. 2a edición. p. 205.

Kawulich, B. (2005) La observación participante como método de recolección de datos. (c) 2005 FQS http://www.qualitative-research. net/fqs/ Forum Qualitative Sozialforschung / Forum: Qualitative 
Social Research (ISSN 1438-5627)

Lacan, J. (2002). Seminario 3, Las Psicosis. Barcelona: Ed. Paidós.

Landazuri, M. (2008), Salir del encierro, medio siglo del Hospital Psiquiátrico San Lázaro, Quito: Editores del Banco Central del Ecuador.

Levi-strauss, C. (2008), Antropología estructural, mito, sociedad, humanidades, México: Editores Siglo XXI.

López Cifuentes, H. (2007) Relato de los tratamientos que se han utilizado en el hospital "San Lázaro" desde su fundación.Febrero-2007 http://www.hpsl.gov.ec/index2. asp?id=202

Marshall, C. \& Rossman, B. (1989). Designing qualitative research. Newbury Park, CA: Sage.

Ministerio de Salud Pública del Ecuador (2014). Plan estratégico de salud mental 2014-2017. Quito, 25 de julio de 2014. Recuperado de: https://aplicaciones.msp.gob.ec/salud/archivosdigitales/documentosDirecciones/dnn/archivos/ ac_00004927_2014\%2025\%20 jul.pdf. [fehca de consulta: 1 ro de abril de 2017]

Parcayacu (1994) "Revista del Instituto Psiquiátrico Sagrado Corazón, número 17

Schensul, Stephen L;; Schensul, Jean J. \& LeCompte, Margaret D. (1999). Essential ethnographic methods: Observations, interviews, and questionnaires (Book 2 en Ethnographer's Toolkit). Walnut Creek, CA: AltaMira Press.Scott, Joan (1996) "El género: una categoría útil para el análisis histórico" 